\title{
Publisher Correction: PAK4 inhibition improves PD-1 blockade immunotherapy
}

Gabriel Abril-Rodriguez, Davis Y. Torrejon, Wei Liu, Jesse M. Zaretsky, Theodore S. Nowicki, Jennifer Tsoi, Cristina Puig-Saus, Ignacio Baselga-Carretero, Egmidio Medina, Michael J. Quist, Alejandro J. Garcia, William Senapedis, Erkan Baloglu, Anusha Kalbasi 10 , Gardenia Cheung-Lau, Beata Berent-Maoz, Begoña Comin-Anduix, Siwen Hu-Lieskovan, Cun-Yu Wang, Catherine S. Grasso and Antoni Ribas (D)

Correction to: Nature Cancer https://doi.org/10.1038/s43018-019-0003-0, published online 9 December 2019.

In the version of this article initially published, the labels above the bottom two plots of Fig. 5e (PAK4 KO isotype (bottom left) and PAK4 KO anti-PD-1 (bottom right)) were incorrect. The correct labels are PAK4 WT isotype (bottom left) and PAK4 WT anti-PD-1 (bottom right). The legend for Fig. 5e also identified one group incorrectly ('B16 PAK4 isotype'). The correct list of groups is 'B16 PAK4 KO isotype; B16 PAK4 KO anti-PD-1; B16 WT isotype; and B16 WT anti-PD-1'. The errors have been corrected in the HTML and PDF versions of the article.

Published online: 22 January 2020

https://doi.org/10.1038/s43018-020-0025-7

(c) The Author(s), under exclusive licence to Springer Nature America, Inc. 2020 